\title{
Statistical Inference for a Simple Constant Stress Model Based on Censored Sampling Data From the Kumaraswamy Weibull Distribution
}

\author{
G. R. AL-Dayian ${ }^{1}$, A. A. EL-Helbawy ${ }^{1} \&$ H. R. Rezk ${ }^{1}$ \\ ${ }^{1}$ Statistics Department, Faculty of Commerce, AL-Azhar University (Girls' Branch), Cairo, Egypt \\ Correspondence: A. A. El-Helbawy, Statistics Department, Faculty of Commerce, AL-Azhar University (Girls' \\ Branch), Cairo, Egypt. E-mail: aah_elhelbawy@hotmail.com
}

Received: March 10, 2014 Accepted: May 30, 2014 Online Published: June 26, 2014

doi:10.5539/ijsp.v3n3p80 URL: http://dx.doi.org/10.5539/ijsp.v3n3p80

\begin{abstract}
In this paper, constant stress accelerated life tests are discussed based on Type I and Type II censored sampling data from Kumaraswmay Weibull distribution. The maximum likelihood estimators are derived for the unknown parameters. The log linear model is assumed as an accelerated model. In addition, confidence intervals for the model parameters are constructed. Optimum test plans, are developed to minimize the generalized asymptotic variance of the maximum likelihood estimators of the model parameters. Monte Carlo simulation is carried out to illustrate the theoretical results of the maximum likelihood estimates, confidence intervals and optimum test plans.
\end{abstract}

Keywords: accelerated life tests, constant stress, Type I censoring, Type II censoring, Kumaraswmay Weibull distribution, confidence intervals, optimum test plans, generalized asymptotic variance, Monte Carlo simulation

\section{Introduction}

The customers expectations are having high quality reliable products on time and at competitive prices. Therefore, experimenters use accelerated life testing (ALT) which enables them to quickly reduce the testing time and save a lot of manpower, material sources and money.

Stress can be applied in different ways but the commonly used types are constant stress, step stress and progressive stress. In ALT, units are subjected to a more severe environment (for instance, very high levels of voltage, excessive vibrations, excessive force, ... etc.) than usual conditions to obtain failures more quickly. Constant stress accelerated test allows each unit in the experiment to run at a prespecified constant stress level. A sample size $n$ is divided into $n_{1}, n_{2}, \ldots, n_{k}$, where $n=\sum_{j=1}^{k} n_{j}$. It is assumed that $n_{1}$ units are all run under a constant stress $x_{1}, n_{2}$ under a constant stress $x_{2}, \ldots$, and $n_{k}$ under a constant stress $x_{k}$. It is assumed that $x_{1}<x_{2}<\ldots<x_{k}$. Determining a relationship between stress and one parameter of the lifetime distribution, extrapolation to the normal working conditions will be carried out. This relationship is known as the acceleration model. It is assumed that changing the stress from one level to another affects the value of the parameter only and not the functional form of the lifetime distribution. This is a major assumption of accelerated life testing. Some references in the field of the ALT include Lydersen and Rausand (1987), Nelson (1990), Drop et al. (1996), Bagdonavicius and Nikulin (2002), Mousa and Jaheen (2002), Murthy et al. (2004), Wu and Yu (2005), Wang (2006, 2009, 2010), Abd-Elfattah et al. (2008), Balakrishnan and Han (2008), Wu et al. (2008), Abdel-Hamid (2009), Abdel-Hamid and AL-Hussaini (2009), Li and Fard (2009), Han and Balakrishnan (2010), Ismail (2011), Bakoban (2012), Hassan and AL-Thobety (2012), and Mitra et al. (2013).

Kumaraswamy (Kum) distribution is applicable to many natural phenomena whose outcomes have lower and upper bounds, such as heights of individuals, scores obtained in a test, atmospheric temperatures and hydrological data. Also, Kum distribution could be appropriate in situations where scientists use probability distributions which have infinite lower and or upper bounds to fit data, when in reality the bounds are finite. Cordeiro et al. (2010) indicated that the Kumaraswamy Weibull (KumW) distribution provides significantly better fits than all of its important submodels (Weibull, exponentiated Weibull and exponentiated exponential) for at least two data sets. This distribution 
is a general case of some important distributions such as a new Kumaraswamy exponential, a new Kumaraswamy Rayleigh, exponentiated exponential, exponentiated Weibull, exponentiated Rayleigh, Weibull, Rayleigh and exponential distributions. The KumW has three shape parameters. These parameters allow for a high degree of flexibility. It attracts wider applications in reliability, engineering and in other areas of research. If there are such models, four parameter models sharing all these features, or if such models are developed in the future then their performance will have to be assessed in terms of hazard rates and the ability to model a variety of data sets. Cordeiro et al. (2010) derive the explicit expressions for the moments and moment generating function, the moments of the order statistics, the asymptotic distributions of the extreme values, explicit expressions for the mean deviations, Bonferroni and Lorenz curves, reliability and Renyi entropy.

This paper deals with KumW distribution as a composite distribution since generation of cumulative distribution functions by composition with other cumulative distributions or functions of such distributions could add an extra parameter to a distribution. Adding a parameter or more to a distribution makes it more flexible to fitting data, see AL-Hussaini (2012). The KumW has not been applied under censoring and ALT in all the previous literature. The objective of this paper is to obtain the maximum likelihood estimators for the unknown parameters for a constant stress ALT based on Type I and Type II censored sample data from KumW distribution. Also, confidence intervals for the parameters are constructed and optimum test plans are developed.

The rest of this paper is organized as follows. Section 2 presents a brief summary about the KumW distribution as a composite distribution. The constant stress life testing based on Type I censoring inference is discussed in Section 3, while in Section 4, the statistical inference for constant stress life testing based on Type II censoring is obtained.

\section{The Kumaraswamy Weibull Distribution}

Kumaraswamy (1980) constructed a distribution with two shape parameters on $(0,1)$. The Kum cumulative distribution function $(\mathrm{CDF})$ is defined by

$$
\mathrm{H}(\mathrm{y}) \equiv H(y ; \theta, \beta)=1-\left(1-y^{\theta}\right)^{\beta}, \quad y \in(0,1),
$$

where $\theta, \beta>0$ are shape parameters.

A composition of $H$, given by (1) and a CDF G, with positive support, yields a new CDF, given by

$$
F(t)=H[G(t)]=1-\left[1-(G(t))^{\theta}\right]^{\beta} .
$$

The composite function $F$ is known as Kum-G distribution, see Jones (2009). On composition of distribution functions, see AL-Hussaini (2012). In particular, if $G$ is Weibull $(\lambda, \phi)$, with CDF, given by

$$
G(t) \equiv G(t ; \lambda, \phi)=1-\exp \left(-(\lambda t)^{\phi}\right), t>0,(\lambda, \phi>0),
$$

then, from (2) and (3), the $\mathrm{CDF}$ of $\mathrm{KumW}(\lambda, \phi, \beta, \theta)$ distribution is given by

$$
F(t) \equiv F(t ; \lambda, \phi, \beta, \theta)=1-\left[1-\left(1-\exp \left(-(\lambda t)^{\phi}\right)\right)^{\theta}\right]^{\beta} .
$$

The probability distribution function (PDF) corresponding to (4) is given by

$$
\begin{aligned}
& f(t ; \theta, \beta, \phi, \lambda)=\theta \beta \phi \lambda^{\phi} t^{\phi-1} \exp \left(-(\lambda t)^{\phi}\right)\left[1-\exp \left(-(\lambda t)^{\phi}\right)\right]^{\theta-1} \quad\left[1-\left[1-\exp \left(-(\lambda t)^{\phi}\right)\right]^{\theta}\right]^{\beta-1}, \\
& t>0, \beta, \theta, \lambda, \phi>0,
\end{aligned}
$$

where $\theta, \beta$ and $\phi$ are the shape parameters and $\lambda$ is a scale parameter.

Figure 1, describes the PDF of KumW distribution for different values of parameters. 

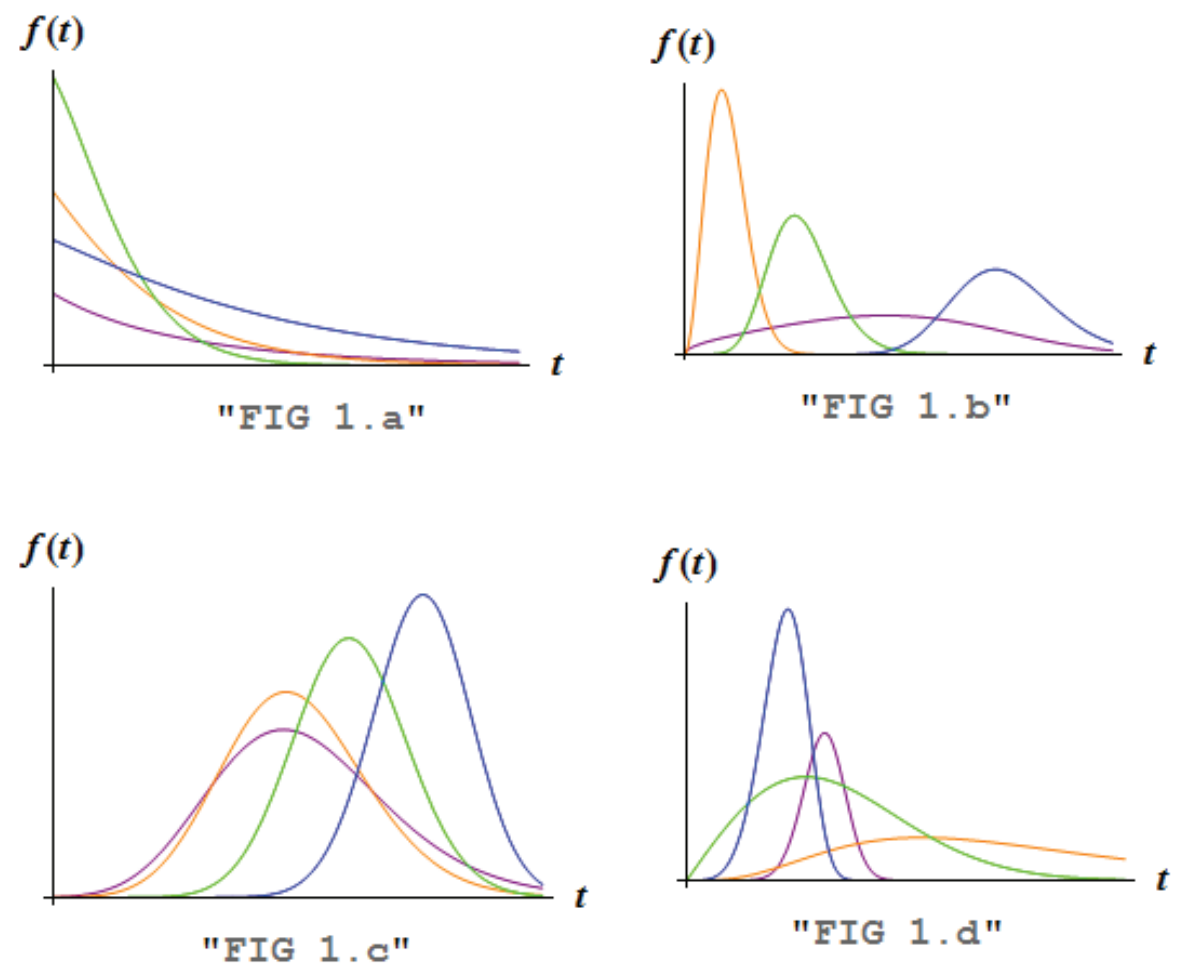

Figure 1. Density functions of KumW distribution

In FIG 1.a, at $\theta=0.5,1.5,2,4, \beta=0.8,1,2,5, \phi, \lambda=1,1,1,1$, the KumW density is monotonically decreasing. In FIG 1.b, $\theta=0.5,2,10,70, \beta=0.5,1.2,3.5,5, \phi=3,2,1.1,1, \lambda=0.4,2,1.3,1$, it has unimodel curve. FIG 1.c, at $\theta=40,50,100,200, \beta=3,5,10,17, \phi, \lambda=1,1,1,1$, has curves approximately symmetric while in FIG $1 . d$, $\theta=5,1,1,5, \beta=1,1,5,5, \phi=1,1,5,3, \lambda=1,1,1,1$, the curves are positive skewed.

The reliability function (rf) of KumW is

$$
R(t ; \theta, \beta, \phi, \lambda)=\left[1-\left[1-\exp \left(-(\lambda t)^{\phi}\right)\right]^{\theta}\right]^{\beta}, \quad t>0 .
$$

The hazard rate function (hrf) of KumW is given as follows:

$$
h(t ; \theta, \beta, \phi, \lambda)=\frac{\theta \beta \phi \lambda^{\phi} t^{\phi-1} \exp \left(-(\lambda t)^{\phi}\right)\left[1-\exp \left(-(\lambda t)^{\phi}\right)\right]^{\theta-1}}{1-\left[1-\exp \left(-(\lambda t)^{\phi}\right)\right]^{\theta}}, \quad t>0 .
$$

The KumW distribution has three shape parameters $\theta, \beta$ and $\phi$. The three shape parameters allow high degree for flexibility of the KumW distribution. The KumW hrf does not involve any complicated function and it can be easily computed numerically. Moreover, it is quite flexible for modeling survival data. Figure 2, shows the hazard rate function of KumW distribution for different values of parameters. 

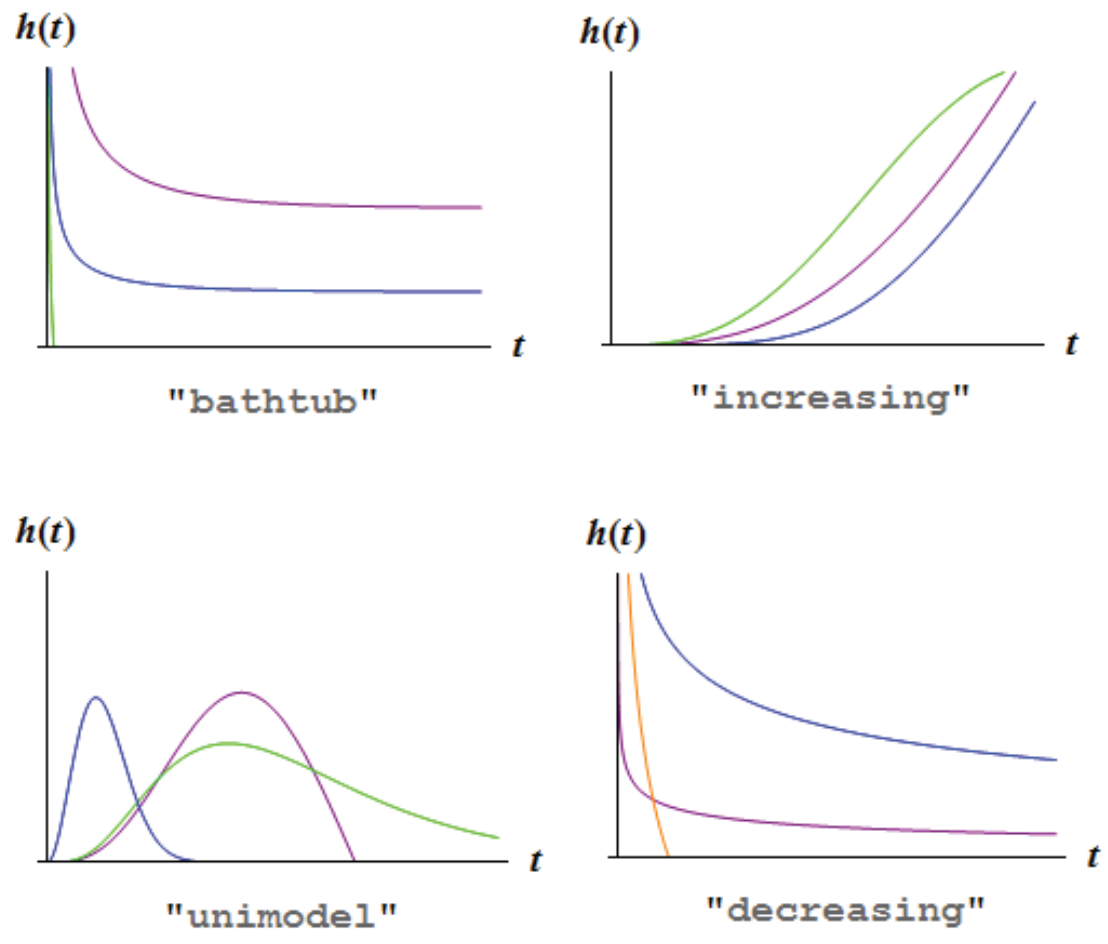

Figure 2. The hrf's of KumW distribution for different values of parameters

The hrf is bathtub at $\theta=0.2,0.1,0.3, \beta=2,0.5,0.5, \phi=1,1,0.2, \lambda=1,1,3$. The hrf is increasing at $\theta=5,4,3$, $\beta=1.2,3,2, \phi=1,1,1, \lambda=1,2,3$. At $\theta=10,5,7, \beta=10,12,15, \phi=0.5,0.6,0.7, \lambda=10,8,3$, the hrf is unimodel while at $\theta=1.5,2,0.5, \beta=1.9,1.2,2.1, \phi=0.5,0.7,0.9, \lambda=0.2,2,2$, the hrf is decreasing. The KumW distribution represents most major hazard shapes: constant, increasing, decreasing, bathtub and unimodal failure rates.

\section{Inference Based on Type I Censoring}

It is assumed that the stress $\mathrm{x}_{j}$ affects only the shape parameter $\theta_{j}$ of the KumW through a certain acceleration function. The log linear function of stress which is just a simple re-parameterization of the original of the life distribution presented by Abdel-Hamid and AL-Hussaini (2009). That is $\log \left(\theta_{j}\right)=a+b x_{j}$ where $a$ and $b$ are unknown parameters depending on the nature of the product and the test method. In the present section, the estimation problem of the parameters of the KumW distribution in constant accelerated life testing based on Type I censored data is discussed. In addition, confidence intervals for the model parameters are constructed. Optimum test plans, that determine the best choice of the proportion of test unit allocated to each stress, are developed. Such optimum test plans minimize the generalized asymptotic variance (GAV) of the maximum likelihood estimators (MLEs) of the model parameters. Numerical study is presented to illustrate the theoretical results.

\subsection{Maximum Likelihood Estimation Based on Type I Censoring}

Let the lifetime experiment be assumed under $\mathrm{k}$ levels of high stress $x_{j}, j=1,2, \ldots, k$ and assume that $x_{u}$ is the usual condition such that $x_{u}<x_{1}<\ldots<x_{k}$. Also, $n_{j}$ units are put on test at each $x_{j}, j=1,2, \ldots, k$. When a Type I censoring is applied at each stress level the lifetime at stress $x_{j}, t_{i j}, i=1,2, \ldots, n_{j}, j=1,2, \ldots, k$ is assumed to be realized from KumW distribution with PDF

$$
\begin{aligned}
f\left(t_{i j} ; \beta, \phi, \lambda, \theta_{j}\right)= & \theta_{j} \beta \phi \lambda^{\phi} t_{i j}^{\phi-1} \exp \left(-\left(\lambda t_{i j}\right)^{\phi}\right) \times\left[1-\exp \left(-\left(\lambda t_{i j}\right)^{\phi}\right)\right]^{\theta_{j}-1} \\
& \times\left[1-\left[1-\exp \left(-\left(\lambda t_{i j}\right)^{\phi}\right)\right]^{\theta_{j}}\right]^{(\beta-1)}
\end{aligned}
$$

where $t_{i j}>0, \beta, \theta, \lambda, \phi>0, j=1,2, \ldots, k$ and $i=1,2, \ldots, n_{j}$. The stress $x_{j}$ affects only the shape parameter of KumW distribution $\theta_{j}$ through a certain acceleration model. Applying Type I censoring at each stress level, the experiment terminates once all the items fail or when a fixed censoring time $t_{c j}$ is reached. The corresponding 
likelihood function is expressed as follows:

$$
\begin{aligned}
L_{1}(\beta, \phi, \lambda, \underline{\theta} ; \underline{t})= & \prod_{j=1 i=1}^{k} \prod_{j}^{n_{j}}\left[\theta_{j} \beta \phi \lambda^{\phi} t_{i j}^{\phi-1} \exp \left(-\left(\lambda t_{i j}\right)^{\phi}\right)\left[g^{\theta_{j}-1}\left(t_{i j} ; \lambda, \phi\right)\right]\right. \\
& \left.*\left[1-g^{\theta_{j}}\left(t_{i j} ; \lambda, \phi\right)\right]^{\beta-1}\right]^{\delta_{i j}}\left[1-g^{\theta_{j}}\left(t_{i j} ; \lambda, \phi\right)\right]^{\beta\left(1-\delta_{i j}\right)},
\end{aligned}
$$

where $\delta_{i j}$ is an indicator variable such that:

$$
\delta_{i j}=\left\{\begin{array}{lll}
1 & \text { for } & t_{i j} \leq t_{c j} \\
0 & \text { for } & t_{i j}>t_{c j}
\end{array},\right.
$$

and

$$
\begin{aligned}
\theta_{j} & =\exp \left(a+b x_{j}\right), \\
g\left(t_{i j} ; \lambda, \phi\right) & =1-\exp \left(-\left(\lambda t_{i j}\right)^{\phi}\right), \\
\left(1-g\left(t_{i j} ; \lambda, \phi\right)\right) & =\exp \left(-\left(\lambda t_{i j}\right)^{\phi}\right), \\
g^{\theta_{j}}\left(t_{i j} ; \lambda, \phi\right) & =\left[1-\exp \left(-\left(\lambda t_{i j}\right)^{\phi}\right)\right]^{\exp \left(a+b x_{j}\right)} .
\end{aligned}
$$

The ML estimator of $a, b, \beta, \phi$ and $\lambda$ are obtained by maximizing the logarithm of the likelihood function, denoted by $\ell_{1}$ which can be written in the form:

$$
\begin{aligned}
\ell_{1}=\ln L\left(t_{i j} ; \beta, \phi, \lambda, \theta_{j}\right)= & \sum_{j=1}^{k} \sum_{i=1}^{n_{j}} \delta_{i j} \ln \left(\theta_{j}\right)+\sum_{j=1}^{k} \sum_{i=1}^{n_{j}} \delta_{i j} \ln (\beta) \\
& +\sum_{j=1}^{k} \sum_{i=1}^{n_{j}} \delta_{i j} \ln (\phi)+\phi \sum_{j=1}^{k} \sum_{i=1}^{n_{j}} \delta_{i j} \ln (\lambda)+(\phi-1) \sum_{j=1}^{k} \sum_{i=1}^{n_{j}} \delta_{i j} \ln \left(t_{i j}\right) \\
& -\sum_{j=1}^{k} \sum_{i=1}^{n_{j}} \delta_{i j}\left(\lambda t_{i j}\right)^{\phi}+\sum_{j=1}^{k}\left(\theta_{j}-1\right) \sum_{i=1}^{n_{j}} \delta_{i j} \ln \left(g\left(t_{i j} ; \lambda, \phi\right)\right) \\
& +(\beta-1) \sum_{j=1}^{k} \sum_{i=1}^{n_{j}} \delta_{i j} \ln \left[1-g^{\theta_{j}}\left(t_{i j} ; \lambda, \phi\right)\right] .
\end{aligned}
$$

The MLEs are found by setting the partial first derivatives of $\ell_{1}$ with respect to $\mathrm{a}, \mathrm{b}, \beta, \phi$ and $\lambda$, respectively, to zeros. A system of non-linear equations can be solved numerically using the Newton-Raphson method, to obtain the MLEs $\hat{a}_{1}, \hat{b}_{1}, \hat{\beta}_{1}, \hat{\phi}_{1}$ and $\hat{\lambda}_{1}$. The MLEs $\hat{a}_{1}, \hat{b}_{1}, \hat{\beta}_{1}, \hat{\phi}_{1}$ and $\hat{\lambda}_{1}$ have an asymptotic variance-covariance matrix defined by inverting the information matrix. Because of the invariance property of MLE, the value of the shape parameter $\theta_{u}$, under stress $x_{u}$, and the MLEs of the rf and hrf under usual conditions at mission time $t_{0}$ could be estimated. The MLEs of the shape parameter of KumW distribution, $\theta_{u}$, can be derived using the following equation:

$$
\hat{\theta}_{1 u}=\exp \left(\hat{a}_{1}+\hat{b}_{1} x_{u}\right) .
$$

Also, the MLEs of the rf and the hrf under usual conditions at a mission time $t_{0}$ are derived using the invariance of MLEs based on (6) and (7), respectively.

The asymptotic variance-covariance matrix of the estimators $\hat{a}_{1}, \hat{b}_{1}, \hat{\beta}_{1}, \hat{\phi}_{1}$ and $\hat{\lambda}_{1}$ are obtained depending on the inverse asymptotic Fisher information matrix $\tilde{I}_{1}$ using the second derivatives of the logarithm of the likelihood function.

The asymptotic Fisher information matrix can be written as follows:

$$
\tilde{I}_{1}=-\left[\frac{\partial^{2} \ell_{1}}{\partial \psi_{i} \partial \psi_{j}}\right], i, j=1,2, \ldots, 5,
$$

where $\psi_{1}=a, \psi_{2}=b, \psi_{3}=\beta, \psi_{4}=\phi$ and $\psi_{5}=\lambda$.

\subsection{The Asymptotic Confidence Intervals Based on Type I Censoring}

For large sample size, the MLEs under appropriate regularity conditions are consistent and asymptotically unbiased as well as asymptotically normally distributed. Therefore, the two sided approximate $100(1-\alpha) \%$ confidence 
intervals for the MLE say, $\hat{w}$ of a population value $\mathrm{w}$ can be obtained by $\mathbf{P}\left(-z \leq \frac{\hat{w}-w}{\sigma_{\hat{w}}} \leq z\right)=(1-\alpha)$ where $\mathrm{z}$ is the $100\left(1-\frac{\alpha}{2}\right)$ th standard normal percentile. The two sided approximate $100(1-\alpha) \%$ confidence intervals for $w$, will be given as follows:

$$
L_{w}=\hat{w}-z_{\frac{\alpha}{2}} \hat{\sigma}_{\hat{w}} \quad \text { and } \quad \mathrm{U}_{w}=\hat{w}+z_{\frac{\alpha}{2}} \hat{\sigma}_{\hat{w}}
$$

where $\hat{\sigma}_{\hat{w}}$ is the standard deviation, in this study $\hat{w}$ is $\hat{a}, \hat{b}, \hat{\beta}, \hat{\phi}$ or $\hat{\lambda}$, respectively, see Nelson (1982).

\subsection{Optimum Test Plans Based on Type I Censoring}

Planning optimal constant sress ALT using maximum likelihood method has been the subject of many studies. Given a known sample size, the objective is usually to find the optimal stress changing time that minimizes the GAV of the MLEs of the model parameters, which is the reciprocal of the determinant of the asymptotic Fisher information matrix $\tilde{I}_{1}$ (see Wu et al., 2008).

That is

$$
\operatorname{GAV}\left(\hat{a}_{1}, \hat{b}_{1}, \hat{\beta}_{1}, \hat{\phi}_{1}, \hat{\lambda}_{1}\right)=\left|\tilde{I}_{1}\right|^{-1} .
$$

Thus, minimization of the GAV is equivalent to maximization of the determinant of $\tilde{I}_{1}$. In case of Type I, the Newton-Raphson method is applied to determine numerically the best choice of the censoring time at each level of stress which minimizes the GAV. Accordingly, the corresponding optimal censoring time at each level of stress can be obtained by getting the first partial derivatives of $\left|\tilde{I}_{1}\right|$ with respect to $t_{c 1}$ and $t_{c 2}$ and then equating to zero,

$$
\frac{\partial\left|\tilde{I}_{1}\right|}{\partial t_{c j}}, j=1,2
$$

where $t_{c j}$ are the censoring times. The determinant can be obtained as follows

$$
\left|\tilde{I}_{1}\right|=\sum_{s=1}^{5} A_{s},
$$

where the elements of determinant are shown in the appendix.

\subsection{Numerical Results}

This section aims to illustrate the theoretical results of both estimation and optimal design problems on the basis of simulated data.

\subsubsection{Simulation Algorithm}

- Several data sets are generated from KumW distribution for a combination of the population parameter values of $a, b, \beta, \phi$ and $\lambda$. Also, for samples of size (20,60 and 100) using 1000 replications for each sample size.

- The transformation between uniform distribution and KumW distribution is given as follows

$$
U_{i, j}=1-\left[1-\left[1-\exp \left(-\left(\lambda t_{i, j}\right)^{\phi}\right)\right]^{\theta_{j}}\right]^{\beta}
$$

- It is assumed that only two different levels of stress, $(k=2), x_{1}=1$ and $x_{2}=1.5$, which are higher than the stress at usual condition, $x_{u}=0.5$.

- The pre-specified censoring times are $t_{c 1}=2$ and $t_{c 2}=3.65$.

- The population parameter values of $a, b, \beta, \phi$ and $\lambda$ used in this simulation study are $(0.5,1.5,1.2,2,2)$.

- A computer program is derived depending on MathCad 14 using the iterative technique of Newton Raphson method to solve the derived nonlinear logarithmic likelihood equation simultaneously.

- Once the values of $\hat{a}_{1}, \hat{b}_{1}, \hat{\beta}_{1}, \hat{\phi}_{1}$ and $\hat{\lambda}_{1}$ are obtained, these estimates are used to estimate, depending on (13) and the design stress, $x_{u}=0.5$, the shape parameter under this stress. $\theta_{u}$, is estimated as $\hat{\theta}_{1 u}=\exp \left(\hat{a}_{1}+\hat{b}_{1} x_{u}\right)$. Also, the rf and the hrf are estimated for different values of mission times under usual condition.

- Evaluating the performance of the estimators of $a, b, \beta, \phi$ and $\lambda$ has been considered through some measurements of accuracy. In order to study the precision and variation of MLEs, it is convenient to use the relative absolute bias $\left(\mathrm{RAB}_{1}\right)=\frac{\text { estimate-population parameter| }}{\text { population parameter }}$, the mean square error $\left(\mathrm{ER}_{1}\right)$ and the relative error $\left(\mathrm{RE}_{1}\right)=\frac{\sqrt{\mathrm{MSE}(\text { estimate) }}}{\text { population parameter }}$. 
- The central asymptotic confidence intervals are obtained for the five parameters $a, b, \beta, \phi$ and $\lambda$, using (15). The results are displayed in Tables 1-4.

Table 1. The $E_{1}, \mathrm{RAB}_{1}, \mathrm{ER}_{1}$ and $\mathrm{RE}_{1}$ of the estimates for 20,60 and 100 sample sizes

\begin{tabular}{cccccc}
\hline $\mathrm{n}$ & parameter & $\mathrm{E}_{1}$ & $\mathrm{RAB}_{1}$ & $\mathrm{ER}_{1}$ & $\mathrm{RE}_{1}$ \\
\hline 20 & $\mathrm{a}$ & 0.4581 & 0.0838 & 0.2128 & 0.9226 \\
& $\mathrm{~b}$ & 1.2830 & 0.1447 & 0.3471 & 0.3928 \\
& $\beta$ & 1.7135 & 0.4279 & 0.4637 & 0.5675 \\
& $\phi$ & 2.3289 & 0.16445 & 0.3584 & 0.2993 \\
& $\lambda$ & 2.2324 & 0.1162 & 0.2548 & 0.2524 \\
\hline 60 & $\mathrm{a}$ & 0.4725 & 0.0550 & 0.1608 & 0.8019 \\
& $\mathrm{~b}$ & 1.4103 & 0.0598 & 0.1746 & 0.2786 \\
& $\beta$ & 1.3214 & 0.1012 & 0.1739 & 0.3475 \\
& $\phi$ & 2.2490 & 0.1245 & 0.2120 & 0.2302 \\
& $\lambda$ & 2.1804 & 0.0902 & 0.1925 & 0.2194 \\
\hline 100 & $\mathrm{a}$ & 0.4935 & 0.0130 & .01604 & 0.2533 \\
& $\mathrm{~b}$ & 1.4170 & 0.0553 & 0.0249 & 0.1050 \\
& $\beta$ & 1.2609 & 0.0507 & 0.0197 & 0.1170 \\
& $\phi$ & 2.1536 & 0.0768 & 0.0406 & 0.10075 \\
& $\lambda$ & 2.1388 & 0.0694 & 0.0363 & 0.0952 \\
\hline
\end{tabular}

Table 2. The estimated shape parameter, reliability function and hazard rate function under usual condition at 20, 60 and 100 sample sizes

\begin{tabular}{ccccccc}
\hline $\mathrm{n}$ & $\hat{\theta}_{1 u}$ & $\mathrm{t}_{0}$ & $\hat{\mathrm{R}}_{1 u}\left(\mathrm{t}_{0}\right)$ & $\mathrm{RAB}_{1 R}$ & $\hat{\mathrm{h}}_{1 u}\left(\mathrm{t}_{0}\right)$ & $\mathrm{RAB}_{1 h}$ \\
\hline 20 & 3.0030 & 0.3 & 0.9421 & 0.0403 & 1.1557 & 2.1915 \\
& & 0.5 & 0.4395 & 0.4240 & 7.2221 & 1.9315 \\
& & 0.7 & 0.0467 & 0.8644 & 15.1494 & 1.7528 \\
& & 1 & 0.0009 & 0.9973 & 25.860 & 1.7569 \\
\hline 60 & 3.2467 & 0.3 & 0.9676 & 0.0142 & 0.6655 & 0.8378 \\
& & 0.5 & 0.6023 & 0.2107 & 4.6278 & 0.8785 \\
& & 0.7 & 0.1385 & 0.5977 & 10.0403 & 0.8244 \\
& & 1 & 0.0023 & 0.9360 & 170.095 & 0.8225 \\
\hline 100 & 3.3268 & 0.3 & 0.9718 & 0.0099 & 0.5660 & 0.5631 \\
& & 0.5 & 0.6560 & 0.1403 & 3.8093 & 0.5462 \\
& & 0.7 & 0.1963 & 0.4301 & 8.2354 & 0.4965 \\
& & 1 & 0.0069 & 0.8076 & 13.8653 & 0.4782 \\
\hline
\end{tabular}

Table 3. Asymptotic confidence bounds of the parameters at confidence level $95 \%$ for 20, 60 and 100 sample sizes

\begin{tabular}{ccccccc}
\hline $\mathrm{n}$ & parameter & $\mathrm{E}_{1}$ & $\mathrm{SE}_{1}$ & $\mathrm{U}_{1}$ & $\mathrm{~L}_{1}$ & length \\
\hline 20 & $\mathrm{a}$ & 0.4581 & 0.4613 & 0.7063 & 0.2937 & 0.4126 \\
& $\mathrm{~b}$ & 1.2830 & 0.5892 & 1.5465 & 1.0195 & 0.5270 \\
& $\beta$ & 1.7135 & 0.6810 & 2.0180 & 1.4090 & 0.6091 \\
& $\phi$ & 2.3289 & 0.5948 & 2.5966 & 2.0612 & 0.5355 \\
& $\lambda$ & 2.2324 & 0.5048 & 2.4581 & 2.0067 & 0.4515 \\
\hline 60 & $\mathrm{a}$ & 0.4725 & 0.4010 & 0.6189 & 0.3261 & 0.2928 \\
& $\mathrm{~b}$ & 1.4103 & 0.4179 & 1.5629 & 1.2577 & 0.3052 \\
& $\beta$ & 1.3214 & 0.4170 & 1.4737 & 1.1691 & 0.3045 \\
& $\phi$ & 2.2490 & 0.4604 & 2.4171 & 2.0809 & 0.3363 \\
& $\lambda$ & 2.1804 & 0.4387 & 2.3406 & 2.0202 & 0.3204 \\
\hline 100 & $\mathrm{a}$ & 0.4935 & 0.1266 & 0.5397 & 0.4473 & 0.0925 \\
& $\mathrm{~b}$ & 1.4170 & 0.1575 & 1.4746 & 1.3594 & 0.1152 \\
& $\beta$ & 1.2609 & 0.1404 & 1.3122 & 1.2096 & 0.1025 \\
& $\phi$ & 2.1536 & 0.2015 & 2.2272 & 2.0800 & 0.1472 \\
& $\lambda$ & 2.1388 & 0.1904 & 2.2083 & 2.0693 & 0.1391 \\
\hline
\end{tabular}


Table 4. The results of optimal design of the life test for 20, 60 and 100 sample sizes

\begin{tabular}{cccccc}
\hline $\mathrm{n}$ & $\bar{r}_{1}$ & $\bar{r}_{1}$ & $t_{c 1}^{*}$ & $t_{c 2}^{*}$ & $\mathrm{GAV}$ \\
\hline 20 & 7 & 7 & 1.243 & 2.745 & 0.0924 \\
60 & 23 & 23 & 3.729 & 8.235 & 0.000728 \\
100 & 37 & 37 & 6.215 & 13.725 & 0.0000982 \\
\hline
\end{tabular}

\subsubsection{Concluding Remarks}

- It is clear from Table 1 that the MLEs $\left(E_{1}\right)$ improve by increasing the sample size. Also, as shown in the numerical results the $\mathrm{RAB}_{1}, \mathrm{ER}_{1}$ and $\mathrm{RE}_{1}$ are decreasing when the sample size is increasing. In addition, the experiment at ALT is rapidly finished more than at usual conditions.

- Table 2 indicates that the reliability decreases when the mission time $t_{0}$ increases. The results get better in the sense that the aim of an accelerated life testing experiments is to get large number of failures (reduce the reliability) of the device with high reliability. In other words, when sample size increases, the rf increases. Also, the RAB $1 R$ for the rf decreases when the sample size increases. The hrf increases when the mission time $\mathrm{t}_{0}$ increases.

- The two-sided $95 \%$ central asymptotic confidence intervals for the parameters of KumW are displayed in Table 3. This table contains the standard error $\left(\mathrm{SE}_{1}\right)$, lower bound $\left(\mathrm{L}_{1}\right)$, upper bound $\left(\mathrm{U}_{1}\right)$ and the length of intervals. The interval estimate of the parameter becomes narrower as the sample size increases.

- It can be observed from the numerical results presented in Table 4 that the optimum test plans do not specify the same censoring time to each stress. Also, Table 4, includes the optimal censoring time at each level of stress for the considered different sample sizes represented by $t_{c 1}^{*}$ and $t_{c 2}^{*}$ which minimizes the GAV of the MLE of the model parameters. As indicated from the results. The optimal GAV of the MLE of the model parameters is decreasing as the sample size $n$ is increasing.

\section{Inference Based on Type II Censoring}

In this section, the estimation problem of the parameters of the KumW distribution in constant accelerated life testing based on Type II censored data is discussed. In addition, central confidence intervals for the model parameters are constructed. Optimum test plans are developed. For illustration, numerical study is presented.

\subsection{Maximum Likelihood Estimation Based on Type II Censoring}

Assuming that the life testing experiment is considered under k levels of high stress $x_{j}, j=1,2, \ldots, k$ and $x_{u}$ is the usual condition such that $x_{u}<x_{1}<x_{2}<\ldots<x_{k}$, at each $x_{j}, j=1,2, \ldots, k$, where $n_{j}$ are items put on test. When a Type II censoring is adopted at each stress level, the experiment terminates once the number of failures $r_{j}$ out of items $n_{j}$ are reached. The lifetime at stress $x_{j}, t_{i j}, i=1,2, \ldots, r_{j}, j=1,2, \ldots, k$, is assumed to follow the KumW distribution with the PDF defined in (5).

The likelihood function based on Type II censoring according to constant stress can be written as:

$$
L_{2}(\beta, \phi, \lambda, \underline{\theta} ; \underline{\mathrm{t}})=\prod_{j=1}^{k} \frac{n_{j} !}{\left(n_{j}-r_{j}\right) !} \prod_{i=1}^{r_{j}} f\left(t_{i j}\right)\left[R\left(t_{r j}\right)\right]^{n_{j}-r_{j}},
$$

where $t_{i j}>0, \beta, \theta, \lambda, \phi>0, j=1,2, \ldots, k$ and $i=1,2, \ldots, r_{j}$. The stress $x_{j}$ affects only the shape parameter of KumW distribution $\theta_{j}$ through a certain acceleration model.

The likelihood function of the experiment takes the following form:

$$
\begin{aligned}
L_{2}(\beta, \phi, \lambda, \underline{\theta} ; \underline{\mathrm{t}})= & \prod_{j=1}^{k} \frac{n_{j} !}{\left(n_{j}-r_{j}\right) !} \prod_{i=1}^{r_{j}} \theta_{j} \beta \phi \lambda^{\phi} t_{i j}^{\phi-1}\left(1-\mathrm{g}\left(t_{i j} ; \lambda, \phi\right)\right)\left(g^{\theta_{j}-1}\left(t_{i j} ; \lambda, \phi\right)\right) \\
& {\left[1-g^{\theta_{j}}\left(t_{i j} ; \lambda, \phi\right)\right]^{\beta-1}\left[\left[1-g^{\theta_{j}}\left(t_{r j} ; \lambda, \phi\right)\right]^{\beta\left(n_{j}-r_{j}\right)}\right], }
\end{aligned}
$$

where $\theta_{j}, \mathrm{~g}\left(t_{i j} ; \lambda, \phi\right)$ and $\mathrm{g}^{\theta_{j}}\left(t_{i j} ; \lambda, \phi\right)$ are given in (11).

$$
g\left(t_{r j} ; \lambda, \phi\right)=1-\exp \left(-\left(\lambda t_{r j}\right)^{\phi}\right)
$$

The MLEs of $a, b, \beta, \phi$ and $\lambda$ are obtained by maximizing the logarithm of (21), denoted by $\ell_{2}$ and can be written 
as follows:

$$
\begin{aligned}
\ell_{2}=\ln L_{2}(\beta, \phi, \lambda, \underline{\theta} ; \underline{\mathrm{t}}) \propto & \sum_{j=1}^{k} r_{j} \ln \left(\theta_{j}\right)+\sum_{j=1}^{k} r_{j} \ln \beta+\sum_{j=1}^{k} r_{j} \ln \phi \\
& +\phi \sum_{j=1}^{k} r_{j} \ln \lambda+(\phi-1) \sum_{j=1}^{k} \sum_{i=1}^{r_{j}} \ln \left(t_{i j}\right) \\
& -\sum_{j=1}^{k} \sum_{i=1}^{r_{j}}\left(\lambda t_{i j}\right)^{\phi}+\sum_{j=1}^{k}\left[\theta_{j}-1\right] \sum_{i=1}^{r_{j}} \ln \left(g\left(t_{i j} ; \lambda, \phi\right)\right) \\
& +(\beta-1) \sum_{j=1}^{k} \sum_{i=1}^{r_{j}} \ln \left(1-g^{\theta_{j}}\left(t_{i j} ; \lambda, \phi\right)\right) \\
& +\beta \sum_{j=1}^{k}\left(n_{j}-r_{j}\right) \sum_{i=1}^{r_{j}} \ln \left(1-g^{\theta_{j}}\left(t_{r j} ; \lambda, \phi\right)\right) .
\end{aligned}
$$

The MLEs can be found by setting the partial first derivatives of $\ell_{2}$ to zero. A system of non-linear equations can be solved numerically using the Newton-Raphson method, to obtain the MLEs $\hat{a}_{2}, \hat{b}_{2}, \hat{\beta}_{2}, \hat{\phi}_{2}$ and $\hat{\lambda}_{2}$. The MLEs $\hat{a}_{2}, \hat{b}_{2}$, $\hat{\beta}_{2}, \hat{\phi}_{2}$ and $\hat{\lambda}_{2}$ have an asymptotic variance covariance matrix defined by inverting the information matrix. Because of the invariance property of MLE, the value of the shape parameter $\theta_{u}$, and the MLE of the rf and hrf under usual conditions at mission time $t_{0}$ could be estimated. The MLE of the shape parameter of KumW distribution, $\theta_{u}$ can be derived using the following equation:

$$
\hat{\theta}_{2 u}=\exp \left(\hat{a}_{2}+\hat{b}_{2} x_{u}\right) .
$$

Also, the MLEs of the rf and the hrf under usual conditions at a mission time $t_{0}$ are derived using the invariance of MLEs based on (6) and (7), respectively.

The asymptotic variance-covariance matrix of the estimators $\hat{a}_{2}, \hat{b}_{2}, \hat{\beta}_{2}, \hat{\phi}_{2}$ and $\hat{\lambda}_{2}$ are obtained depending on the inverse asymptotic Fisher information matrix $\tilde{I}_{2}$ using the second derivatives of the logarithm of the likelihood function.

The asymptotic Fisher information matrix can be written as follows:

$$
\tilde{I}_{2}=-\left[\frac{\partial^{2} \ell_{2}}{\partial \omega_{i} \partial \omega_{j}}\right], i, j=1,2, \ldots, 5,
$$

where $\omega_{1}=a, \omega_{2}=b, \omega_{3}=\beta, \omega_{4}=\phi$ and $\omega_{5}=\lambda$.

\subsection{Optimum Test Plans Based on Type II Censoring}

Statistically, the larger the sample size is the better results-more accurate estimation can be achieved. on the other side, from the producer's point of view, coducting test with a large sample size will take up a long time and capital. Therefore, it is significant to gain an appropriate sample size to balance these two sides. The GAV of the MLEs of the model parameters is the reciprocal of the determinant of the asymptotic Fisher information matrix $\tilde{I}_{2}$ (see $\mathrm{Wu}$ et al., 2008).

That is

$$
\operatorname{GAV}\left(\hat{a}_{2}, \hat{b}_{2}, \hat{\beta}_{2}, \hat{\phi}_{2}, \hat{\lambda}_{2}\right)=\left|\tilde{I}_{2}\right|^{-1} .
$$

Thus, minimization of the GAV is equivalent to maximization of the determinant of $\tilde{I}_{2}$. Newton Raphson method is applied to determine numerically the best choice of the sub sample proportion allocated to each level of stress which minimizes the GAV as defined previously. Accordingly, the corresponding optimal numbers of items allocated to each level of stress can be obtained by getting the first partial derivatives of $\left|\tilde{I}_{2}\right|$ with respect to $p_{1}$ and $p_{2}$ then equating to zero,

$$
\frac{\partial\left|\tilde{I}_{2}\right|}{\partial p_{j}}, j=1,2 .
$$

where $p_{j}$ are the sub sample proportion. The determinant can be obtained as follows

$$
\left|\tilde{I}_{2}\right|=\sum_{s=1}^{5} B_{s},
$$

and the results are shown in the appendix. 
Remark 1 When $r=n$ all the results obtained for Type II censoring reduce to the complete sample case.

\subsection{Numerical Results}

This section aims to illustrate the theoretical results of both estimation and optimal design problems on basis of simulated and real data.

\subsubsection{Simulation Algorithm}

The same steps of the algorithm in Subsection (3.4.1), will be considered in this algorithm with the following data:

- Numbers of test units are allocated to each level of stress $\left(n_{j}, j=1,2\right)$ where $p_{1}=0.5, p_{2}=0.5, r_{j}=0.90\left(n_{j}\right)$.

- The population parameter values of $a, b, \beta, \phi$ and $\lambda$ used in this simulation study are $(0.5,1.5,1.2,2,2)$ to generate $t_{i j}, j=1,2$ and $i=1,2, \ldots, r_{j}$.

- The nonlinear logarithmic likelihood equation is solved simultaneously.

- Once the values of $\hat{a}_{2}, \hat{b}_{2}, \hat{\beta}_{2}, \hat{\phi}_{2}$ and $\hat{\lambda}_{2}$ are obtained, these estimates and the design stress, $x_{u}=0.5$ are used to estimate the shape parameter under this stress. $\theta_{u}$, is estimated as $\hat{\theta}_{2 u}=\exp \left(\hat{a}_{2}+\hat{b}_{2} x_{u}\right)$. Also, the rf and the hrf are estimated for different values of mission times under usual conditions.

- The performance of $\hat{a}_{2}, \hat{b}_{2}, \hat{\beta}_{2}, \hat{\phi}_{2}$ and $\hat{\lambda}_{2}$ has been evaluated through some measurements of accuracy. In order to study the precision and variation of MLEs, it is convenient to use the relative absolute bias $\left(\mathrm{RAB}_{2}\right)$, the mean square error $\left(\mathrm{ER}_{2}\right)$ and the relative error $\left(\mathrm{RE}_{2}\right)$.

- The two sided approximate $100(1-\alpha) \%$ central asymptotic confidence intervals for $a, b, \beta, \phi$ and $\lambda$ are obtained through using steps analogous to those used in (15) noting that $\hat{w}$ denotes $\hat{a}_{2}, \hat{b}_{2}, \hat{\beta}_{2}, \hat{\phi}_{2}$ and $\hat{\lambda}_{2}$ respectively. The results are displayed in Tables 5-8.

Table 5. The $\mathrm{E}_{2}, \mathrm{RAB}_{2}, \mathrm{ER}_{2}$ and $\mathrm{RE}_{2}$ of the estimates for 20,60 and 100 sample sizes

\begin{tabular}{cccccc}
\hline $\mathrm{n}$ & parameter & $\mathrm{E}_{2}$ & $\mathrm{RAB}_{2}$ & $\mathrm{ER}_{2}$ & $\mathrm{RE}_{2}$ \\
\hline 20 & $\mathrm{a}$ & 0.4563 & 0.0874 & 0.2129 & 0.9228 \\
& $\mathrm{~b}$ & 1.7829 & 0.1886 & 0.3470 & 0.3927 \\
& $\beta$ & 0.9503 & 0.2081 & 0.1724 & 0.3460 \\
& $\phi$ & 2.3476 & 0.1738 & 0.2308 & 0.2402 \\
& $\lambda$ & 1.7195 & 0.1403 & 0.2897 & 0.2691 \\
\hline 60 & $\mathrm{a}$ & 0.4862 & 0.0276 & 0.0612 & 0.4948 \\
& $\mathrm{~b}$ & 1.6395 & 0.0930 & 0.1195 & 0.2305 \\
& $\beta$ & 1.1624 & 0.0313 & 0.1114 & 0.2781 \\
& $\phi$ & 2.1894 & 0.0947 & 0.1489 & 0.1929 \\
& $\lambda$ & 1.9361 & 0.0321 & 0.1150 & 0.1696 \\
\hline 100 & $\mathrm{a}$ & 0.4937 & 0.0126 & 0.0130 & 0.2280 \\
& $\mathrm{~b}$ & 1.5392 & 0.0261 & 0.0369 & 0.1280 \\
& $\beta$ & 1.1874 & 0.0105 & 0.0158 & 0.1258 \\
& $\phi$ & 2.1160 & 0.0580 & 0.0522 & 0.2285 \\
& $\lambda$ & 1.9553 & 0.02235 & 0.0158 & 0.0628 \\
\hline
\end{tabular}


Table 6. The estimated shape parameter and reliability function under usual condition at 20, 60 and 100 sample sizes

\begin{tabular}{ccccccc}
\hline $\mathrm{n}$ & $\hat{\theta}_{2 u}$ & $\mathrm{t}_{0}$ & $\hat{\mathrm{R}}_{2 u}\left(\mathrm{t}_{0}\right)$ & $\mathrm{RAB}_{2 R}$ & $\hat{\mathrm{h}}_{2 u}\left(\mathrm{t}_{0}\right)$ & $\mathrm{RAB}_{2 h}$ \\
\hline 20 & 3.8488 & 0.3 & 0.9984 & 0.0171 & 0.0436 & 0.8795 \\
& & 0.5 & 0.9318 & 0.2211 & 0.9140 & 0.6290 \\
& & 0.7 & 0.6181 & 0.7948 & 3.3862 & 0.3847 \\
& & 1 & 0.1165 & 2.2469 & 7.6426 & 0.1852 \\
\hline 60 & 3.6912 & 0.3 & 0.9917 & 0.0103 & 0.1934 & 0.4659 \\
& & 0.5 & 0.8195 & 0.0738 & 2.1255 & 0.1372 \\
& & 0.7 & 0.3790 & 0.1005 & 5.6769 & 0.0316 \\
& & 1 & 0.0320 & 0.1086 & 10.6028 & 0.1304 \\
\hline 100 & 3.5371 & 0.3 & 0.9875 & 0.0067 & 0.2682 & 0.2593 \\
& & 0.5 & 0.7916 & 0.0373 & 2.3121 & 0.0615 \\
& & 0.7 & 0.3583 & 0.0405 & 5.6659 & 0.0296 \\
& & 1 & 0.0324 & 0.0984 & 10.1708 & 0.0843 \\
\hline
\end{tabular}

Table 7. Asymptotic confidence bounds of the parameters at confidence level $95 \%$ for 20, 60 and 100 sample sizes

\begin{tabular}{ccccccc}
\hline $\mathrm{n}$ & parameter & $\mathrm{E}_{2}$ & $\mathrm{SE}_{2}$ & $\mathrm{U}_{2}$ & $\mathrm{~L}_{2}$ & length \\
\hline 20 & $\mathrm{a}$ & 0.4563 & 0.4614 & 0.6626 & 0.2500 & 0.4127 \\
& $\mathrm{~b}$ & 1.7829 & 0.5891 & 2.0463 & 1.5195 & 0.5269 \\
& $\beta$ & 0.9503 & 0.4152 & 1.1360 & 0.7646 & 0.3714 \\
& $\phi$ & 2.3476 & 0.4804 & 2.5624 & 2.1328 & 0.4297 \\
& $\lambda$ & 1.7195 & 0.5382 & 1.9602 & 1.4788 & 0.4814 \\
\hline 60 & $\mathrm{a}$ & 0.4862 & 0.2474 & 0.5501 & 0.4223 & 0.1277 \\
& $\mathrm{~b}$ & 1.6395 & 0.3457 & 1.7288 & 1.5502 & 0.1785 \\
& $\beta$ & 1.1624 & 0.3338 & 1.2486 & 1.0762 & 0.1724 \\
& $\phi$ & 2.1894 & 0.3859 & 2.2890 & 2.0898 & 0.1993 \\
& $\lambda$ & 1.9361 & 0.3391 & 2.0237 & 1.8485 & 0.1751 \\
\hline 100 & $\mathrm{a}$ & 0.4937 & 0.1140 & 0.5165 & 0.4709 & 0.0456 \\
& $\mathrm{~b}$ & 1.5392 & 0.1921 & 1.5776 & 1.5008 & 0.0768 \\
& $\beta$ & 1.1874 & 0.1887 & 1.2251 & 1.1497 & 0.0755 \\
& $\phi$ & 2.1160 & 0.1257 & 1.9804 & 1.9302 & 0.0503 \\
& $\lambda$ & 1.9553 & 0.2285 & 2.1617 & 2.0703 & 0.0914 \\
\hline
\end{tabular}

Table 8. The results of optimal design of the life test for 20, 60 and 100 sample sizes

\begin{tabular}{ccccccc}
\hline $\mathrm{n}$ & $\mathrm{n}_{1}$ & $\mathrm{n}_{2}$ & $\mathrm{p}_{1}$ & $\mathrm{n}_{1}^{*}$ & $\mathrm{n}_{2}^{*}$ & $\mathrm{GAV}$ \\
\hline 20 & 10 & 10 & 0.4218 & 8 & 12 & 0.00625 \\
60 & 30 & 30 & 0.4319 & 26 & 34 & 0.000291 \\
100 & 50 & 50 & 0.4515 & 45 & 55 & 0.0000147 \\
\hline
\end{tabular}

\subsubsection{Concluding Remarks}

- It is clear from Table 5 that the MLEs $\left(\mathrm{E}_{2}\right)$ improve by increasing the sample size. Also, as shown in the numerical results the $\mathrm{RAB}_{2}, \mathrm{ER}_{2}$ and $\mathrm{RE}_{2}$ are decreasing when the sample size is increasing. The number of failures at ALT is rapidly obtained more than at usual conditions.

- Table 6 indicates that the rf decreases when the mission time $t_{0}$ increases. The results get better in the sense that the aim of an accelerated life testing experiments is to get large number of failures (reduce the rf) of the device with high reliability. In other words, when sample size increases, the rf increases. Also, the RAB $\mathrm{AB}_{2}$ the $\mathrm{rf}$ decreases when the sample size increases. The hrf increases when the mission time $t_{0}$ increases.

- The two-sided 95\% central asymptotic confidence intervals for the parameters of KumW are given in Table 7. This table contains the standard error $\left(\mathrm{SE}_{2}\right)$, lower bound $\left(\mathrm{L}_{2}\right)$, upper bound $\left(\mathrm{U}_{2}\right)$ and the length of intervals. The interval estimate of the parameters becomes narrower as the sample size increases. 
- Optimum test plans are developed numerically. The expected number of items that must be allocated to each level of stress represented by $n_{1}^{\star}, n_{2}^{\star}$ which minimizes the GAV is displayed in Table 8 . As indicated from the results, the optimal GAV of the MLE of the model parameters decreases as the sample size $\mathrm{n}$ increases.

\subsubsection{Application}

This subsection aims to demonstrate how the proposed method can be used in practice. Cordeiro et al. (2010) used Kolmogorov Smirnov goodness of fit test and data points representing failure time. The data were taken from Murthy et al. (2004). The data were 30 items $(n=30)$ tested with test stopped after 20th failure $(r=20)$. It is assumed that $k=2$, i.e. there are only two different levels of stress $x_{1}=0.6$ and $x_{2}=1$, which are higher than the stress at usual conditions, $x_{u}=0.5$. The failure times in the first level are $[0.0014,0.0623,1.3826,2.0130,2.5274$, $2.8221,3.1544,4.9835,5.5462,5.8196]$ and the failure times in the second level of stress are [ 5.8714, 7.4710, $7.5080,7.6667,8.6122,9.0442,9.1153,9.6477,10.1547,10.7582]$

The initial values of $a, b, \beta, \phi$ and $\lambda$ used in this application are $a=0.5, b=1.5, \beta=2, \phi=2$ and $\lambda=2$. Once the estimate value of $a, b, \beta, \phi$ and $\lambda$ are obtained, the estimates are used to estimate and the design stress, $x_{u}=0.5$, the shape parameter under this stress. $\theta_{u}$ is estimated, $\hat{\theta}_{2 u}=\exp \left(\hat{a}_{2}+\hat{b}_{2} x_{u}\right)$. Also, the rf and the hrf are estimated for different values of mission times under usual conditions.

The estimated shape parameter, rf and hrf under usual conditions are shown in Table 9. It shows that the rf decreases when the mission time increases while the hrf increases when the mission time increases. The relationship between the stress and the shape parameter is tested through testing the significance of the coefficient $b$. Hypothesis test is obtained when $\alpha=0.05$ and with one degree of freedom, assuming the null hypothesis is $b=0$. It is rejected and the relationship between the level of the stress and the shape parameter exists. Table 10 indicates the central asymptotic confidence intervals of the parameters at confidence level 95\%. The optimal test plan which decreases the GAV $=0.0849$ is at $n^{*}=7$.

Table 9. The estimated shape parameter and reliability function under usual condition

\begin{tabular}{ccccc}
\hline \multicolumn{5}{c}{$\hat{\theta}_{2 u}=4.6147$} \\
\hline $\mathrm{t}_{0}$ & 0.3 & 0.5 & 0.7 & 1 \\
\hline$\hat{\mathrm{R}}_{2 u}\left(\mathrm{t}_{0}\right)$ & 0.9972 & 0.9333 & 0.7013 & 0.2648 \\
$\mathrm{RAB}_{2 R}$ & 0.0159 & 0.2230 & 1.0364 & 6.3799 \\
$\hat{\mathrm{h}}_{2 u}\left(\mathrm{t}_{0}\right)$ & 0.0655 & 0.7539 & 2.1650 & 4.2302 \\
$\mathrm{RAB}_{2 h}$ & 0.8191 & 0.6940 & 0.6066 & 0.5490 \\
\hline
\end{tabular}

Table 10. Estimates and asymptotic confidence bounds of the parameters at confidence level $95 \%$

\begin{tabular}{ccccccc}
\hline parameter & $\mathrm{E}_{2}$ & $\mathrm{RAB}_{2}$ & $\mathrm{SE}_{2}$ & $\mathrm{~L}_{2}$ & $\mathrm{U}_{2}$ & length \\
\hline $\mathrm{a}$ & 0.7293 & 0.4585 & 0.2293 & 0.2707 & 1.1879 & 0.9172 \\
$\mathrm{~b}$ & 1.600 & 0.5440 & 0.2625 & 1.0750 & 2.1250 & 2.0500 \\
$\beta$ & 0.9396 & 0.5302 & 0.2498 & 0.4400 & 1.4392 & 0.9992 \\
$\phi$ & 1.7751 & 0.1125 & 0.1600 & 1.4551 & 2.0951 & 0.6400 \\
$\lambda$ & 1.7995 & 0.1003 & 0.3167 & 1.1661 & 2.4329 & 1.2668 \\
\hline
\end{tabular}

Remark 2 The results obtained in this paper can be modified to obtain special cases results for sub-models of KumW distribution under Type I and Type II censored samples as follows:

- The Kum exponential distribution, if $\phi=1$.

- The Kum Rayleigh distribution, if $\phi=2$.

- The exponentiated Weibull distribution, if $\beta=1$.

- The exponentiated Rayleigh distribution, if $\beta=1$ and $\phi=2$.

- The exponentiated exponential distribution, if $\beta=\phi=1$.

- The Weibull distribution, if $\beta=\theta=1$, see Khamis (1997).

- The Rayleigh distribution, if $\phi=2, \beta=\theta=1$.

- The exponential distribution, if $\phi=\beta=\theta=1$. 


\section{Acknowledgements}

The authors would like to thank Dr. Essam K. AL-Hussaini, Professor of Mathematical Statistics, Alexandria University, for helpful comments and suggestions which led to substantial improvements of this manuscript. Also the authors would like to thank the Referees and the Editor for their comments which led to improvements of an earlier version of this article.

\section{References}

Abdel-Hamid, A. (2009). Constant partially accelerated life tests for Burr Type XII distribution with progressive Type II censoring. Computational Statistics and Data Analysis, 53, 2511-2523. http://dx.doi.org/10.1016/j.csda.2009.01.018

Abdel-Hamid, A., \& AL-Hussaini, E. (2009). Estimation in step stress accelerated life tests for the exponentiated exponential distribution with Type I censoring. Computational Statistics and Data Analysis, 53, 1328-1338. http://dx.doi.org/10.1016/j.csda.2008.11.006

Abd-ELfattah, A., Hassan, A., \& Nassr, S. (2008). Estimation in step stress partially accelerated life tests for the Burr Type XII distribution using Type I censoring. Statistical Methodology, 5, 502-514. http://dx.doi.org/10.1016/j.stamet.2007.12.001

AL-Hussaini, E. K. (2012). Composition of cumulative distribution functions. Journal of Statistical Theory and Applications, 11, 333-336.

Bagdonavicius, V., \& Nikulin, M. (2002). Accelerated Life Models: Modeling and Statistical Analysis. Boca Raton, Florida: Chapman \& Hall-CRC Press.

Bakoban, R. (2012). Estimation in step stress partially accelerated life tests for the generalized inverted exponential distribution using Type I censoring. American Journal of Scientific Research, 3, 25-35.

Balakrishnan, N., \& Han, D. (2008). Exact inference for a simple step stress model with competing risks for failure from exponentail distribution under Type II censoring. Journal of Statistical Planning and Inference, 138, 4172-4186. http://dx.doi.org/10.1016/j.jspi.2008.03.036

Cordeiro, G. M., Ortega, E. M., \& Nadarajah, S. (2010). The Kumaraswamy Weibull distribution with application to failure data. Journal of The Franklin Institute, 347, 1399-1429. http://dx.doi.org/10.1016/j.jfranklin.2010.06.010

Drop, J., Mazzuchi, T., Fornell, G., \& Pollock, L. (1996). A Bayes approach to step-stress accelerated life testing. IEEE Transactions on Reliability, 45, 491-498. http://dx.doi.org/10.1109/24.537021

Han, D., \& Balakrishnan, N. (2010). Inference for a simple step stress model with competing risk for failure from the exponential distribution under time constraint. Computational Statistics and Data Analysis, 54, 2066-2081. http://dx.doi.org/10.1016/j.csda.2010.03.015

Hassan, A., \& AL-Thobety, A. (2012). Optimal design of failure step stress partially accelerated life tests with Type II censored inverted Weibull data. International Journal of Engineering Research and Application, 2, 3242-3253.

Ismail, A. (2011). Planning step stress life tests with Type II censored data. Scientific Research and Essays, 6, 4021-4028.

Jones, M. C. (2009). Kumaraswamy's distribution: a beta type distribution with some tractability advantages. Statistical Methodology, 6, 70-81. http://dx.doi.org/10.1016/j.stamet.2008.04.001

Khamis, I. (1997). Comparison between constant and step stress tests for Weibull models. International Journal of Quality \& Reliability Management, 14, 74-81. http://dx.doi.org/10.1108/02656719710156798

Kumaraswamy, P. (1980). A generalized probability density function for double bounded random processes. Journal of Hydrology, 46, 79-88. http://dx.doi.org/10.1016/0022-1694(80)90036-0

Li, C. H., \& Fard, N. (2009). Optimal simple step stress accelerated life test design for reliability predication. Journal of Statistical Planning and Inference, 139, 1799-1808. http://dx.doi.org/10.1016/j.jspi.2008.05.046

Lydersen, S., \& Rausand, M. (1987). A systematic approach to accelerated life testing. Reliability Engineering, 18, 285-293. http://dx.doi.org/10.1016/0143-8174(87)90033-3 
Mitra, S., Ganguly, A., Samanta, D., \& Kundu, D. (2013). On the simple step stress model for two parameter exponential distribution. Statistical Methodology, 15, 95-114. http://dx.doi.org/10.1016/j.stamet.2013.04.003

Mousa, M., \& Jaheen. M. (2002). Statistical inference for the Burr model based on progressively censored data.

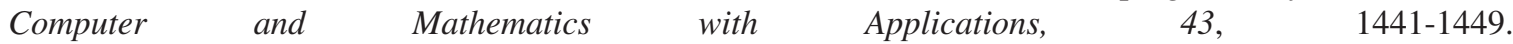
http://dx.doi.org/10.1016/S0898-1221(02)00110-4

Murthy, D., Xie, M., \& Jiang, R. (2004). Weibull models. John Wiley \& Sonc, Inc.

Nelson, W. B. (1982). Applied Life Data Analysis. New York: John Wiley. http://dx.doi.org/10.1002/0471725234

Nelson, W. B. (1990). Accelerated Testing Statistical Models, Test Plans and Data Analysis. John Wiley \& Sonc, Inc.

Wang, B. (2006). Unbiased estimations for the exponential distribution based on step stress accelerated life testing data. Applied Mathematics and Computation, 173, 1227-1237. http://dx.doi.org/10.1016/j.amc.2005.04.066

Wang, B. (2009). Testing for the validity of the assumptions in the exponential step stress accelerated life testing model. Computational Statistics and Data Analysis, 53, 2702-2729. http://dx.doi.org/10.1016/j.csda.2009.01.008

Wang, B. (2010). Interval estimation for exponential progressive Type II censored step stress accelerated life test-

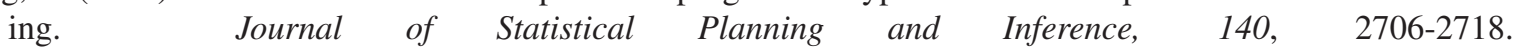
http://dx.doi.org/10.1016/j.jspi.2010.03.033

Wu, J., \& Yu, H. (2005). Statistical inference about the shape parameter of the Burr Type XII distribution under the failure censored sampling plan. Applied Mathematics and Computation, 163, 443-482.

Wu, S., Lin, Y., \& Chen, S. (2008). Optimal step stress test under Type I progressive group censoring with random removals. Journal of Statistical Planning and Inference, 138, 817-826. http://dx.doi.org/10.1016/j.jspi.2007.02.004 


\section{Appendix}

The asymptotic Fisher information matrix in the case of Type I censoring can be rewritten as follows:

$$
\tilde{I}_{1}=-\mathrm{A}_{i j}, \quad i, j=1,2, \ldots, 5 .
$$

The elements of the determinant are given as follows:

$$
\begin{aligned}
& A_{1}=-\left\{A_{11} A_{22} A_{33}\left(A_{44} A_{55}-A_{45}^{2}\right)-A_{11} A_{22} A_{43}\left(A_{43} A_{55}-A_{53} A_{45}\right)\right. \\
& +A_{11} A_{22} A_{35}\left(A_{43} A_{54}-A_{53} A_{44}\right)-A_{11} A_{23}^{2}\left(A_{44} A_{55}-A_{45}^{2}\right) \\
& +A_{11} A_{23} A_{34}\left(A_{42} A_{55}-A_{45} A_{52}\right)-A_{11} A_{23} A_{35}\left(A_{42} A_{54}-A_{44} A_{52}\right) \\
& +A_{11} A_{24} A_{32}\left(A_{43} A_{55}-A_{54} A_{53}\right)-A_{11} A_{24} A_{33}\left(A_{42} A_{55}-A_{45} A_{52}\right) \\
& +A_{11} A_{24} A_{35}\left(A_{42} A_{53}-A_{43} A_{52}\right)-A_{11} A_{25} A_{32}\left(A_{43} A_{54}-A_{44} A_{53}\right) \\
& \left.+A_{11} A_{25} A_{33}\left(A_{42} A_{54}-A_{44} A_{52}\right)-A_{11} A_{25} A_{34}\left(A_{42} A_{53}-A_{43} A_{52}\right)\right\} \text {, } \\
& A_{2}=-\left\{-A_{12}^{2} A_{33}\left(A_{44} A_{55}-A_{45}^{2}\right)+A_{12}^{2} A_{34}\left(A_{43} A_{55}-A_{45} A_{53}\right)\right. \\
& -A_{12}^{2} A_{35}\left(A_{43} A_{54}-A_{44} A_{53}\right)+A_{12} A_{23} A_{31}\left(A_{44} A_{55}-A_{45}^{2}\right) \\
& -A_{12} A_{23} A_{43}\left(A_{41} A_{55}-A_{45} A_{51}\right)+A_{12} A_{23} A_{35}\left(A_{41} A_{54}-A_{44} A_{51}\right) \\
& -A_{12} A_{24} A_{31}\left(A_{43} A_{55}-A_{53} A_{45}\right)+A_{12} A_{24} A_{33}\left(A_{41} A_{55}-A_{45} A_{51}\right) \\
& -A_{12} A_{24} A_{53}\left(A_{41} A_{53}-A_{43} A_{51}\right)+A_{12} A_{25} A_{31}\left(A_{43} A_{54}-A_{44} A_{53}\right) \\
& \left.-A_{12} A_{25} A_{33}\left(A_{41} A_{54}-A_{44} A_{51}\right)+A_{12} A_{25} A_{34}\left(A_{41} A_{53}-A_{43} A_{51}\right)\right\} \text {, } \\
& A_{3}=-\left\{A_{13} A_{12} A_{23}\left(A_{44} A_{55}-A_{45}^{2}\right)-A_{13} A_{21} A_{34}\left(A_{42} A_{55}-A_{45} A_{52}\right)\right. \\
& +A_{13} A_{12} A_{35}\left(A_{42} A_{54}-A_{44} A_{52}\right)-A_{13} A_{22} A_{31}\left(A_{44} A_{55}-A_{45}^{2}\right) \\
& +A_{13} A_{22} A_{34}\left(A_{41} A_{55}-A_{45} A_{51}\right)-A_{13} A_{22} A_{35}\left(A_{41} A_{54}-A_{51} A_{44}\right) \\
& +A_{13} A_{24} A_{31}\left(A_{42} A_{55}-A_{45} A_{54}\right)-A_{13} A_{24} A_{32}\left(A_{41} A_{55}-A_{51} A_{45}\right) \\
& +A_{13} A_{24} A_{35}\left(A_{41} A_{52}-A_{42} A_{51}\right)-A_{13} A_{25} A_{31}\left(A_{42} A_{54}-A_{44} A_{52}\right) \\
& \left.+A_{13} A_{32} A_{25}\left(A_{41} A_{54}-A_{44} A_{51}\right)-A_{13} A_{25} A_{34}\left(A_{41} A_{52}-A_{51} A_{42}\right)\right\} \text {, } \\
& A_{4}=-\left\{-A_{14} A_{12} A_{23}\left(A_{43} A_{55}-A_{45} A_{53}\right)+A_{14} A_{21} A_{33}\left(A_{42} A_{55}-A_{45} A_{52}\right)\right. \\
& -A_{14} A_{12} A_{35}\left(A_{42} A_{53}-A_{43} A_{52}\right)+A_{14} A_{22} A_{31}\left(A_{43} A_{55}-A_{45} A_{53}\right) \\
& -A_{14} A_{22} A_{33}\left(A_{41} A_{55}-A_{45} A_{51}\right)+A_{14} A_{22} A_{35}\left(A_{41} A_{53}-A_{43} A_{51}\right) \\
& -A_{14} A_{23} A_{31}\left(A_{42} A_{55}-A_{45} A_{52}\right)+A_{14} A_{32}^{2}\left(A_{41} A_{55}-A_{51} A_{45}\right) \\
& -A_{14} A_{23} A_{35}\left(A_{41} A_{52}-A_{42} A_{51}\right)+A_{14} A_{25} A_{31}\left(A_{42} A_{53}-A_{43} A_{52}\right) \\
& \left.-A_{14} A_{32} A_{25}\left(A_{41} A_{53}-A_{43} A_{51}\right)+A_{14} A_{25} A_{33}\left(A_{41} A_{52}-A_{51} A_{42}\right)\right\} \text {, }
\end{aligned}
$$

and

$$
\begin{aligned}
A_{5}= & -\left\{A_{15} A_{12} A_{23}\left(A_{43} A_{54}-A_{44} A_{53}\right)-A_{15} A_{21} A_{33}\left(A_{42} A_{54}-A_{44} A_{52}\right)\right. \\
& +A_{15} A_{12} A_{34}\left(A_{42} A_{53}-A_{43} A_{52}\right)-A_{15} A_{22} A_{31}\left(A_{43} A_{54}-A_{44} A_{53}\right) \\
& +A_{15} A_{22} A_{33}\left(A_{41} A_{54}-A_{44} A_{51}\right)-A_{15} A_{22} A_{34}\left(A_{42} A_{53}-A_{43} A_{52}\right) \\
& +A_{15} A_{23} A_{31}\left(A_{42} A_{54}-A_{44} A_{52}\right)-A_{15} A_{32}^{2}\left(A_{41} A_{54}-A_{51} A_{44}\right) \\
& +A_{15} A_{23} A_{34}\left(A_{41} A_{53}-A_{43} A_{51}\right)-A_{15} A_{24} A_{31}\left(A_{42} A_{53}-A_{43} A_{52}\right) \\
& \left.+A_{15} A_{24} A_{32}\left(A_{41} A_{53}-A_{43} A_{51}\right)-A_{15} A_{24} A_{33}\left(A_{41} A_{52}-A_{51} A_{42}\right)\right\} .
\end{aligned}
$$

The asymptotic Fisher information matrix in the case of Type II censoring can be rewritten in short form as follows:

$$
\tilde{I}_{2}=-B_{i j}, \quad i, j=1,2, \ldots, 5,
$$


where the elements of the asymptotic Fisher information matrix in (A.2) will be obtained using steps analogous to those used for obtaining (A.1).

\section{Copyrights}

Copyright for this article is retained by the author(s), with first publication rights granted to the journal.

This is an open-access article distributed under the terms and conditions of the Creative Commons Attribution license (http://creativecommons.org/licenses/by/3.0/). 